\title{
Compact printed ultra-wideband antenna: corrugated monopole augmented with parasitic strips
}

\section{Author names:}

Ming-Chun Tang ${ }^{1,2}$, Zhiyuan Chen ${ }^{1}$, and Richard W. Ziolkowski ${ }^{3,4}$

Author affiliations:

Dr Ming-Chun Tang

1. College of Communication Engineering, Chongqing University, Chongqing 400044, China

2. State Key Laboratory of Millimeter Waves, Nanjing, 210096, China

Mr. Zhiyuan Chen

1. College of Communication Engineering, Chongqing University, Chongqing 400044, China

Prof. Richard W. Ziolkowski

3. Department of Electrical and Computer Engineering, University of Arizona, Tucson, AZ, 85721, USA

4. Global Big Data Technologies Centre, University of Technology Sydney (UTS), Sydney, Australia

Corresponding author: Dr Ming-Chun Tang

E-mail: tangmingchunuestc@126.com

Co-authors:

Mr. Zhiyuan Chen

E-mail: 20151213060@cqu.edu.cn

Prof. Richard W. Ziolkowski

E-mail: ziolkowski@ece.arizona.edu 


\title{
Compact printed ultra-wideband antenna: corrugated monopole augmented with parasitic strips
}

\begin{abstract}
A novel compact microstrip-line-fed ultra-wideband (UWB) antenna is reported in this paper. The antenna consists of a corrugated half-ellipse monopole and three strips that act as near-field resonant parasitic (NFRP) elements. The entire UWB band (3.1-10.6 GHz) is covered from a very compact size: $19 \mathrm{~mm} \times 10 \mathrm{~mm}$. Two of the NFRP strips are utilized to cover more effectively the lower portion of the UWB frequency range; the third one improves the broadside gain values by $3.28 \mathrm{~dB}$ in the upper portion. As a consequence, this compact design maintains a stable radiation performance over the entire UWB band.
\end{abstract}

Keywords: Compact antenna, printed antenna, ultra-wideband, near-field resonant parasitic element

\section{Introduction}

Planar ultra-wideband (UWB) antennas continue to be a topic of high interest because, for instance, their structures are simple and low profile, they are easy to fabricate with low cost, and they are conveniently integrated with monolithic RF circuits [1]. Along with the rapid growth in radio frequency (RF) front-ends over the past few years, there has been a renewed demand for a larger number of microwave devices to be integrated into quite small mobile platforms. Thus, there is an urgency to achieve antennas having even more compact sizes while still providing excellent radiation performance characteristics over the entire UWB operational band. It has stimulated research that dwells deep into the design of compact and high-quality UWB antennas. 
A variety of effective technologies have been implemented to accomplish the desired miniaturization of their physical sizes. These include, for example, etching a half-elliptical slot into the monopole [2], loading the radiator with an inverted L-strip [3], introducing a staircase-shaped radiator [4], combining several metamaterial unit cells [5], and cutting the antenna structure in half [6].

In this paper, a microstrip-line-fed elliptical monopole is cut in half and modified with slots and then integrated with three near-field resonant parasitic (NFRP) strips. The realized design covers the entire UWB band, yet witnesses a $55.3 \%$ reduction in electrical size and improves the broadside realized gain in the upper portion of the UWB frequency range by $3.28 \mathrm{~dB}$ in comparison to its original whole monopole configuration. As a result, the gain values are maintained within a $2.42 \mathrm{~dB}$ variation for all UWB frequencies.

\section{Antenna design and properties}

Figure 1 presents the UWB antenna model and its design parameters. The antenna is built on a FR4 substrate board $\left(\varepsilon_{r}=4.4, \mu_{r}=1.0\right.$, and $\left.\tan \delta=0.02\right)$ with a $1.6 \mathrm{~mm}$ thickness. On the top of the board, a metallic half-ellipse disc is printed with its edge coinciding with one long edge of the board. This radiating patch is fed by a straight microstrip line. As near-field resonant parasitic (NFRP) elements [7], three strips are also printed on this top surface, along the side opposite to the patch. Among these NFRP strips, the two outer most ones have $90^{\circ}$ bends near the top and bottom edges of the substrate. Their presence red shifts the lower bound of the frequencies radiated 
by the half-elliptical patch and, hence, facilitates the antenna miniaturization. The shorter NFRP strip is straight and is arranged nearest to the patch. It increases the broadside gain value in the upper portion of the UWB frequency range. In order to coordinate the capacitive coupling levels between the radiation patch and the three strips, the radiation patch is corrugated by cutting five rectangular strips from it, each with the same width (g). The distances between adjacent corrugations are equal (W5). The presence of these corrugations increases the length of the current pathway on the edges of the radiation patch and, thus, also helps with the antenna miniaturization. The bottom of the board acts as a grounded sheet. A rectangular slot is cut out from it, directly underneath the half-elliptical patch. This feature couples with the patch to help maintain the wideband operation. The optimized values of all of the design parameters are (in millimeters): $\mathrm{L}=19.0, \mathrm{~L} 1=8.7, \mathrm{~L} 2=18.2, \mathrm{~L} 3=2.3, \mathrm{~L} 4=8.3, \mathrm{~L} 5=17.2$, L6=1.7, L7=9.1, L8=2.495, L9=9.1 L10=4.0, W=10.0, W1=0.3, W2=0.2, W3=0.5, $\mathrm{W} 4=2.2, \mathrm{~W} 5=1.2, \mathrm{~W} 6=1.6, \mathrm{~W} 7=4.8, \mathrm{R} 1=5.1, \mathrm{R} 2=3.723$, and $\mathrm{g}=0.3$.

\section{Results and discussion}

The UWB antenna design was simulated with the ANSYS/ANSOFT frequency domain finite element method (FEM)-based high frequency structure simulator (HFSS). It was fabricated and tested. A photograph of the fabricated prototype is shown in Figure 2. The simulated and measured voltage standing wave ratios (VSWRs) are given in Figure 3. For comparison purposes a standard wide-slot elliptical monopole antenna with twice the physical size: $19 \mathrm{~mm} \times 20 \mathrm{~mm}$ was also 
designed and optimized as a reference. Its realized configuration and its simulated VSWR values are also presented in Figure 3 for easy comparison. These results demonstrate that the reference antenna has a wide impedance bandwidth ranging from 3.393 to $11.387 \mathrm{GHz}$ (i.e., those frequencies for which VSWR < 2). In contrast, the simulated (measured) bandwidth of our antenna is from 3.031 (2.916) up to 10.713 (10.735) GHz. It is clear that even with half the physical size, the presence of the corrugations and the NFRP elements red shifts the lower frequency edge of the operating band $\sim 0.362 \mathrm{GHz}$ in the simulated results. This clearly demonstrates that this hybrid technology effectively accomplishes the desired antenna miniaturization. Note that the measured lower frequency bound of the prototype shifted $\sim 0.115 \mathrm{GHz}$ lower than the simulated one. As confirmed by simulations of the fabricated antenna, this is due to the usual unavoidable errors from the fabrication imperfections and the presence of the long coaxial cable used in the measurements. On the whole, the measured values are in good agreement with their simulated counterparts over the entire UWB frequency band.

The far-field radiation performance characteristics were numerically investigated, and the results are reported in Figures 4 and 5. In order to prove the accuracy of the HFSS values, these calculations were repeated with a completely different computational electromagnetics (CEM) approach, i.e., with CST Microwave Studio. They are included for comparison. The agreement between the HFSS and CST values is very good.

One observes in Figure 4 that the E (ZOX)- plane pattern is monopole-like and the $\mathrm{H}$ (ZOY)- plane pattern is omni-directional. Notice that more variations from the monopole and omni patterns are apparent at the higher frequency point, e.g., $9.5 \mathrm{GHz}$. 
These occur because of the presence of higher-order modes as the frequency increases and cause the radiation patterns to become more directional [8]. Figure 5 provides the maximum realized gain values in the broadside direction and in any direction for the prototype and reference antennas. These reference case results clearly indicate that when the frequency goes higher than $10 \mathrm{GHz}$, its maximum gain value is higher than $2.9 \mathrm{dBi}$, while the broadside gain value goes below $0 \mathrm{dBi}$, i.e., the mainbeam direction tends to point significantly away from broadside. This phenomenon is observed in most UWB monopole antenna designs $[9,10]$. In contrast to the reference antenna, the broadside gain values of the reported antenna float in the range of 0.51-2.93 $\mathrm{dBi}$ over the entire UWB band. Clearly, these results demonstrate a significant gain performance improvement in the higher frequency range when compared to the reference case. For example, at $10.6 \mathrm{GHz}$, the broadside gain is improved from -0.68 $\mathrm{dBi}$ to $2.60 \mathrm{dBi}$ (2.77 $\mathrm{dBi}$ in CST), witnessing a $3.28 \mathrm{~dB}(3.45 \mathrm{~dB}$ in CST) enhancement.

In order to clarify the contributions from the presence of the three NFRP strips on the antenna performance characteristics, the simulated surface current distributions on the half-elliptical patch and on these NFRP strips are illustrated in Figure 6 at different frequencies. It is observed that strong currents are located on the outer and middle strips, respectively, at the first two resonant frequencies, i.e., at $3.1475 \mathrm{GHz}$ and 3.2925 GHz. Simulations also demonstrate that these first two resonances arise from the sizes of these two strips and their capacitive coupling to the patch, and that they produce the red-shift of the lower operational frequency bound. At frequencies 
higher than $9 \mathrm{GHz}$, the majority of currents are found, as shown, to be concentrated on the inner NFRP strip, proving that the increase of the broadside gain values in the upper portion of the UWB range is a consequence of the fundamental mode associated with the presence of that inner NFRP strip. Furthermore, the impact of the three NFRP strips on the antenna VSWR was numerically investigated. The results are given in Figure 7 . When loading the main radiator with only the outer (middle) NFRP strip, there is only a single resonant dip located at $3.1475 \mathrm{GHz}(3.2250 \mathrm{GHz})$. On the other hand, when loading it with both the outer and middle NFRP strips, two resonant dips appear at 3.1475 and $3.2925 \mathrm{GHz}$. Their presence contributes to the expansion of the operational frequency range at its lower end. Moreover, with the inner NFRP strip, the impedance match around $9.5 \mathrm{GHz}$ is also improved.

The third resonant dip located at $3.5125 \mathrm{GHz}$ in Figure 6 is ascribed to the fundamental resonance mode of the corrugated half-ellipse monopole element. This frequency value corresponds to the free space wavelength: $\lambda_{0 \text {, monopole }}=85.41 \mathrm{~mm}$. Taking into account the permittivity, the corresponding wavelength in the substrate is $\left.\lambda_{g \text {, monopole }}=\lambda_{0 \text {, monopole }} \Lambda\left(\varepsilon_{\mathrm{r}}+1\right) / 2\right]^{1 / 2}=51.98 \mathrm{~mm}$. The physical length of the corrugated half-ellipse monopole along the $x$-axis in Figure 1 is approximately $19.24 \mathrm{~mm}$, which is a little shorter than a half wavelength. Nonetheless, resonance is achieved because the effective length is a little larger in the presence of the strong capacitive coupling effects between the parasitic elements and the rectangular slot in the ground [11].

As was noted in our antenna design process discussion, several parameters are important when optimizing the impedance matching. In Figure 8, the results from 
three selected parameter variations are given. They provide guidelines for the design and optimization of these types of UWB antennas. As Figure 8(a) indicates, the impedance match over the entire UWB frequency range witnesses variations, except near the first two resonance frequencies, when the feedline strip width (W6) is changed. Figures 8(b) and 8(c) illustrate the impact of varying the slot geometry. While variations also occur over the entire range, the impedance match in the upper end of the operational frequency range changes considerably with variations of the slot length (L9). On the other hand, altering the slot width (W7) changes the coupling levels between the ground and NFRP strips. This effect also causes variations over the entire range, but with a noticeable impact at the lower end. By properly adjusting these slot and strip parameters, one can balance these effects and achieve good impedance matching across the entire UWB band, but particularly at the higher end of the operational frequency range.

Table 1 compares the size and bandwidth of our prototype design to other compact planar UWB antennas recently reported in the literature (Note that the $\left(^{+}\right)$ signs for the data from Ref. [6] mean "beyond the given value” since it did not provide a more accurate upper bound.). The physical and corresponding electrical sizes; the impedance bandwidths (where VSWR $<2$ ); and the corresponding fractional bandwidths are given. It is clearly observed that while our prototype antenna is more compact, it provides a fractional bandwidth that covers the entire UWB frequency range and is comparable to the values achieved with the indicated larger sized antennas. Note that, since the Refs. [2-6] only reported the peak realized gain values 
in any direction, instead of the broadside realized gain values. Thus, the term "NA", not available, is present in the table for those cases.

\section{Conclusion}

The hybrid approach of cutting-in-half the monopole, introducing corrugations on it, and introducing the NFRP elements yielded a novel compact UWB antenna with improved performance characteristics. The antenna was optimized, fabricated and tested. In comparison to a standard wide-slot elliptical monopole UWB antenna, the prototype antenna has a 55.3\% reduction in its electrical size, while the broadside realized gain in the upper portion of the UWB frequency range exhibits a $3.28 \mathrm{~dB}$ improvement. The reported antenna has a compact planar configuration, as well as demonstrated stable radiation performance throughout its operational band, making it an excellent candidate for integration with monolithic RF circuits for numerous UWB applications.

\section{Disclosure statement}

No potential conflict of interest was reported by the authors.

\section{Funding}

This work was supported in part by the National Natural Science Foundation of China contract numbers 61471072, in part by Graduate Scientific Research and Innovation Foundation of Chongqing, China contract number CYB16047, in part by the 
Fundamental Research Funds for the Central Universities contract number 106112015CDJZR165510, in part by China Postdoctoral Science Foundation contract number 2016M590860, and in part by Opening subject of State Key Laboratory of Millimeter Waves contract number K201732.

\section{References}

[1] Adamiuk G, Zwick T, Wiesbeck W. UWB antennas for communication systems. Proc. IEEE 2012; 100: 2308-2321.

[2] $\mathrm{Xu} \mathrm{K}, \mathrm{Zhu} \mathrm{Z,} \mathrm{Li} \mathrm{H,} \mathrm{Huangfu} \mathrm{J,} \mathrm{et} \mathrm{al.} \mathrm{A} \mathrm{printed} \mathrm{single-layer} \mathrm{UWB} \mathrm{monopole}$ antenna with extended ground plane stubs. IEEE Antennas Wirel. Propag. Lett. 2013; 12: 237-240.

[3] Gautam A K, Yadav S, Kanaujia B K. A CPW-fed compact UWB microstrip antenna. IEEE Antennas Wirel. Propag. Lett. 2013; 12: 151-154.

[4] Roshna T K, Deepak U, Sajitha V R, et al. Coplanar stripline-fed compact UWB antenna. Electron. Lett. 2014; 50: 1181-1182.

[5] Islam M M, Islam M T, Samsuzzaman M, et al. Compact metamaterial antenna for UWB applications. Electron. Lett. 2015;51: 1222-1224.

[6] Liu J, Esselle K P, Hay S G, et al. Effects of printed UWB antenna miniaturization on pulse fidelity and pattern stability. IEEE Trans. Antennas Propag. 2014; 62: 3903-3910. 
[7] Ziolkowski R W, Jin P, Lin C -C. Metamaterial-inspired engineering of antennas. Proc. IEEE 2011; 99: 1720-1731.

[8] Tang M -C, Ziolkowski R W, Xiao S. Compact hyper-band printed slot antenna with stable radiation properties. IEEE Trans. Antennas Propag. 2014; 62: 2962 2969.

[9] Li P, Liang J, Chen X. Study of printed elliptical/circular slot antennas for ultrawideband applications. IEEE Trans. Antennas Propag. 2006; 54:1670-1675

[10] Tang M -C, Shi T, Ziolkowski R W. Planar ultra-wideband antennas with improved realized gain performance. IEEE Trans. Antennas Propag. 2016; 64:61-69.

[11] Shao S., Burkholder R. J., Volakis J. L. Design approach for robust UHF RFID tag antennas mounted on a plurality of dielectric surfaces. IEEE Antennas Propag. Mag. 2014; 56:158-166. 
Table 1 Comparisons of our prototype antenna and recently reported compact UWB antennas

\begin{tabular}{|c|c|c|c|c|c|}
\hline References & $\begin{array}{l}\text { Physical } \\
\text { size } \\
(\mathrm{mm} \times \mathrm{mm} \\
\times \mathrm{mm})\end{array}$ & $\begin{array}{l}\text { Electrical size } \\
\left(\lambda_{\mathrm{L}} \times \lambda_{\mathrm{L}} \times \lambda_{\mathrm{L}}\right) \\
\text { where } \lambda_{\mathrm{L}} \text { is the free } \\
\text { space wavelength } \\
\text { corresponding to } \\
\text { the lowest } \\
\text { frequency in the } \\
\text {-10dB bandwidth }\end{array}$ & $\begin{array}{l}\text { Impedance } \\
\text { bandwidth } \\
\text { ( GHz ) }\end{array}$ & $\begin{array}{c}\text { Fractional } \\
\text { bandwidth } \\
\text { ( \% ) }\end{array}$ & $\begin{array}{l}\text { Broadside } \\
\text { realized gain } \\
\text { values at } 10.6 \\
\qquad \mathrm{GHz} \\
(\mathrm{dBi})\end{array}$ \\
\hline Ref.[2] & $24 \times 20 \times 1.0$ & $0.296 \times 0.247 \times 0.012$ & $3.7-10.1$ & 92.75 & NA \\
\hline Ref. [3] & $25 \times 25 \times 1.6$ & $0.217 \times 0.217 \times 0.014$ & $2.6-13.04$ & 133.5 & NA \\
\hline Ref. [4] & $25 \times 7 \times 1.6$ & $0.258 \times 0.072 \times 0.017$ & $3.1-11.4$ & 114.48 & NA \\
\hline Ref. [5] & $\begin{array}{c}27.16 \times 18.9 \\
7 \times 1.6\end{array}$ & $0.278 \times 0.194 \times 0.016$ & $\begin{array}{l}3.07- \\
19.91\end{array}$ & 146.56 & NA \\
\hline Ref. [6] & $\begin{array}{c}17 \times 12 \times 1.5 \\
24\end{array}$ & $0.193 \times 0.136 \times 0.017$ & $\begin{array}{c}3.4- \\
11.0(+)\end{array}$ & $105.56(+)$ & NA \\
\hline Ref. [10] & $\begin{array}{c}37 \times 25 \times 1.5 \\
24\end{array}$ & $0.364 \times 0.246 \times 0.015$ & $\begin{array}{l}2.95- \\
10.82\end{array}$ & 114.31 & $\sim 2.2$ \\
\hline $\begin{array}{l}\text { Prototype } \\
\text { antenna }\end{array}$ & $19 \times 10 \times 1.6$ & $0.185 \times 0.097 \times 0.016$ & $\begin{array}{l}2.92- \\
10.74\end{array}$ & 114.56 & $\begin{array}{rr}2.60 & \text { (HFSS) } \\
2.77 & \text { (CST) }\end{array}$ \\
\hline
\end{tabular}


Figure 1. Configuration and design parameters of the reported UWB antenna.

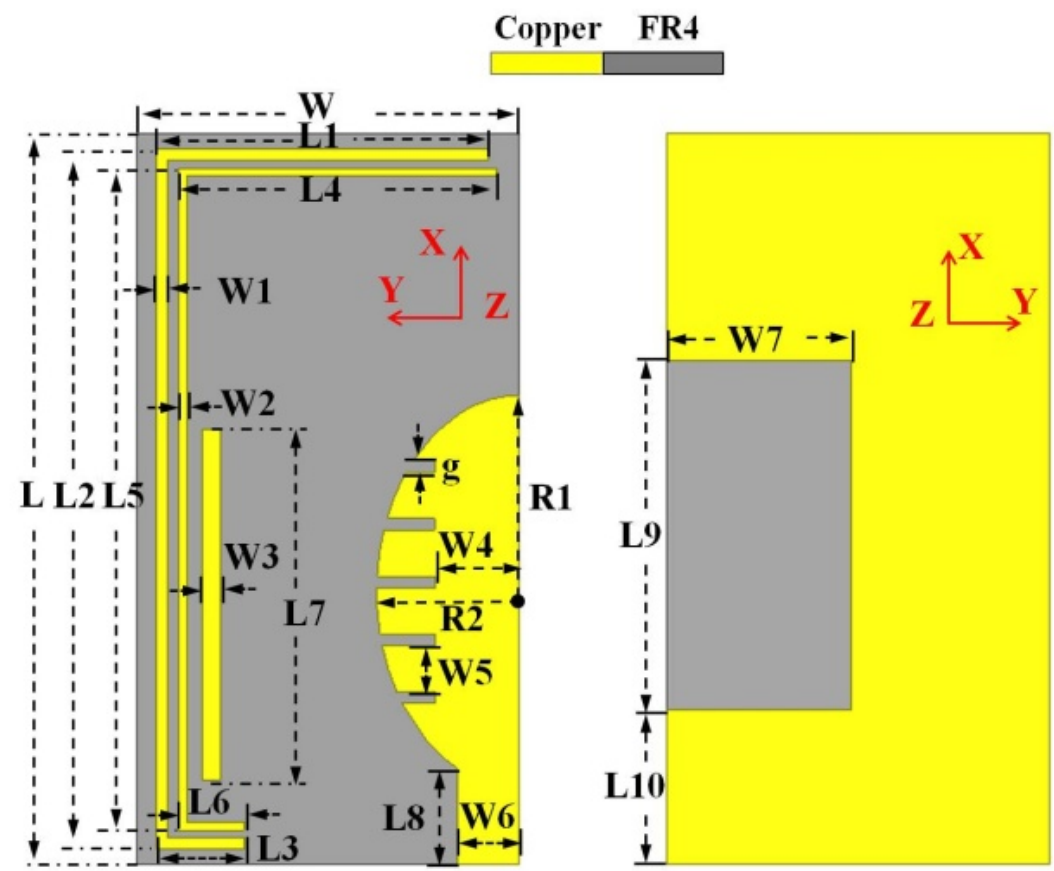

Figure 2. The fabricated prototype of the optimized UWB antenna.

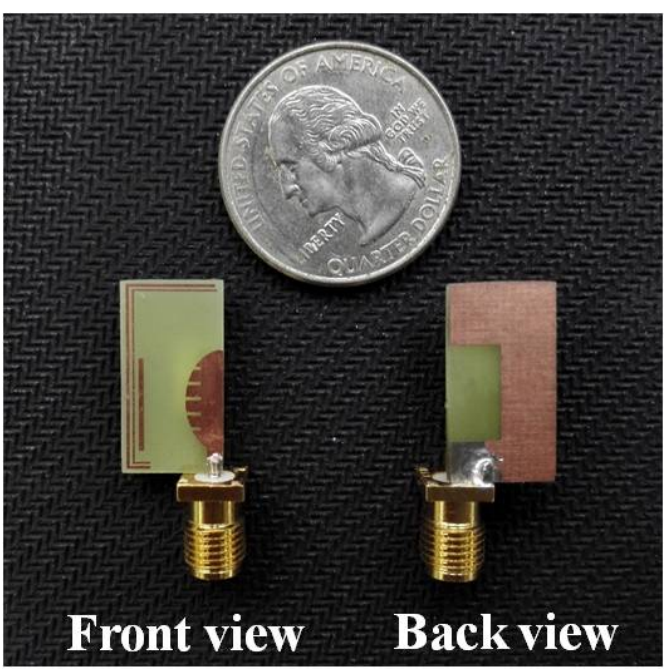


Figure 3. Simulated and measured VSWR values of the prototype antenna and the simulated values of the reference, a standard wide-slot elliptical monopole UWB antenna.

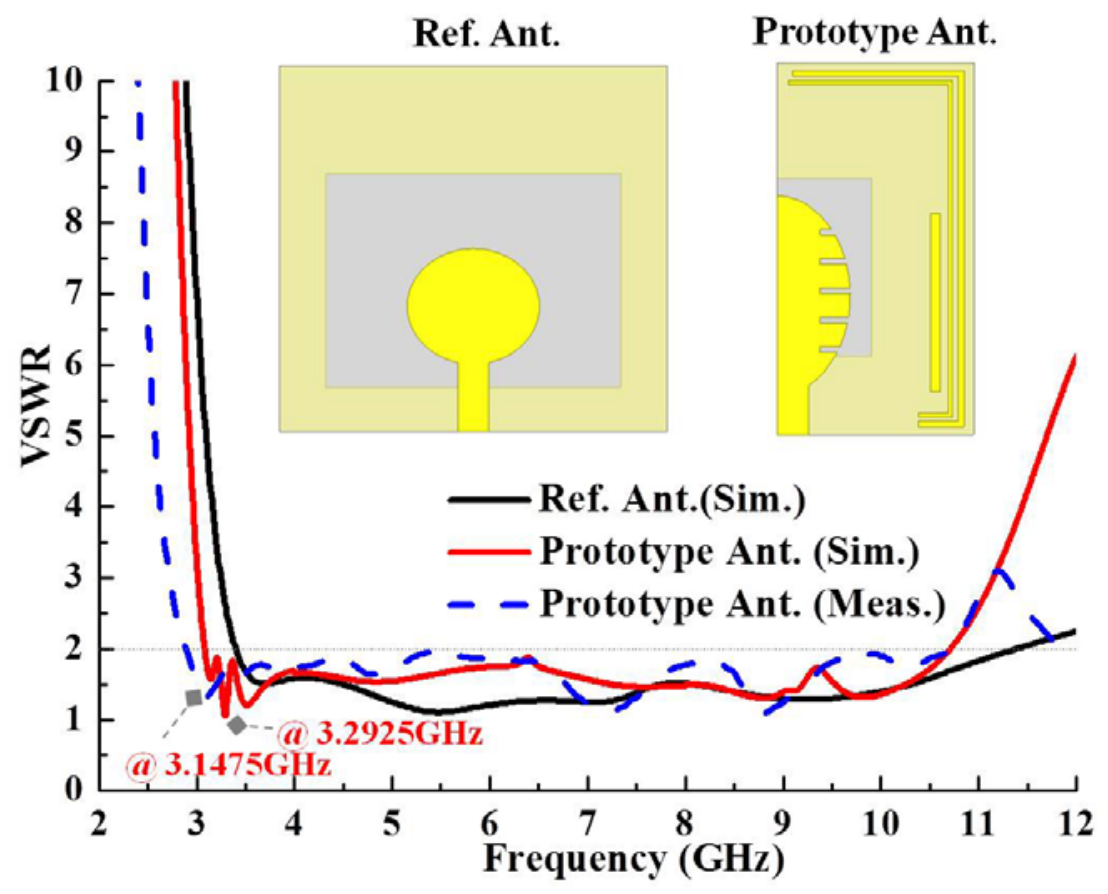


Figure 4. HFSS and CST simulated 2-D realized gain patterns at the frequency points: (a) 3.5, (b) 6.5, and (c) $9.5 \mathrm{GHz}$.

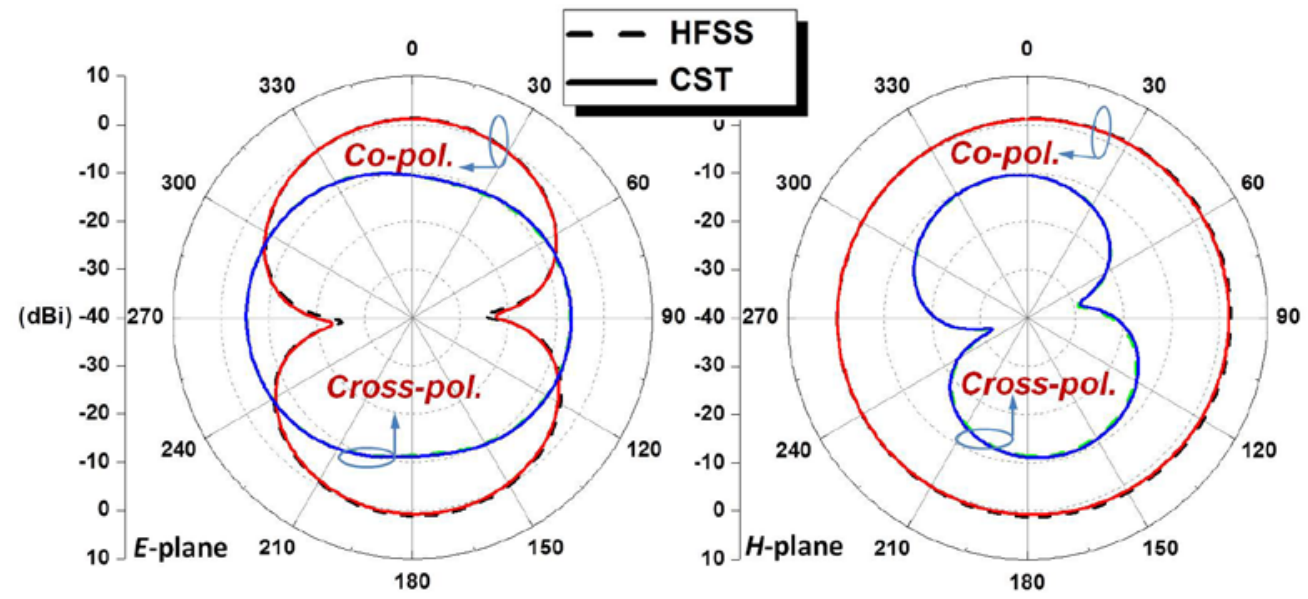

(a)

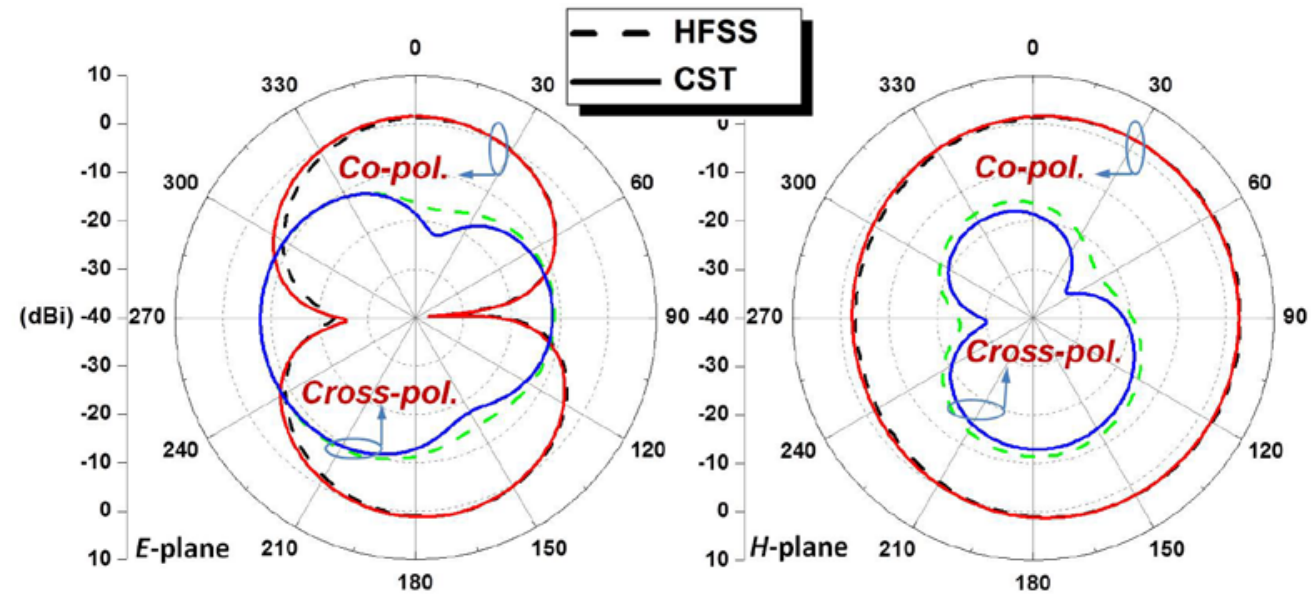

(b)

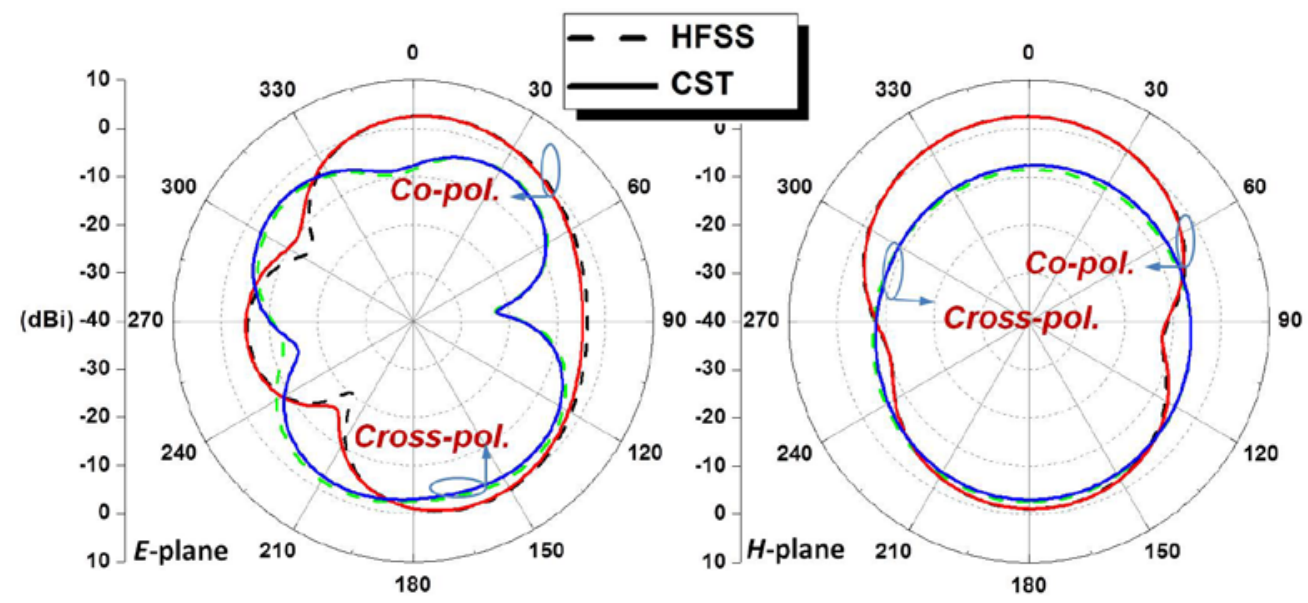

(c) 
Figure 5. The maximum realized gain values in the broadside direction and in any direction for the reference antenna and the prototype antenna.

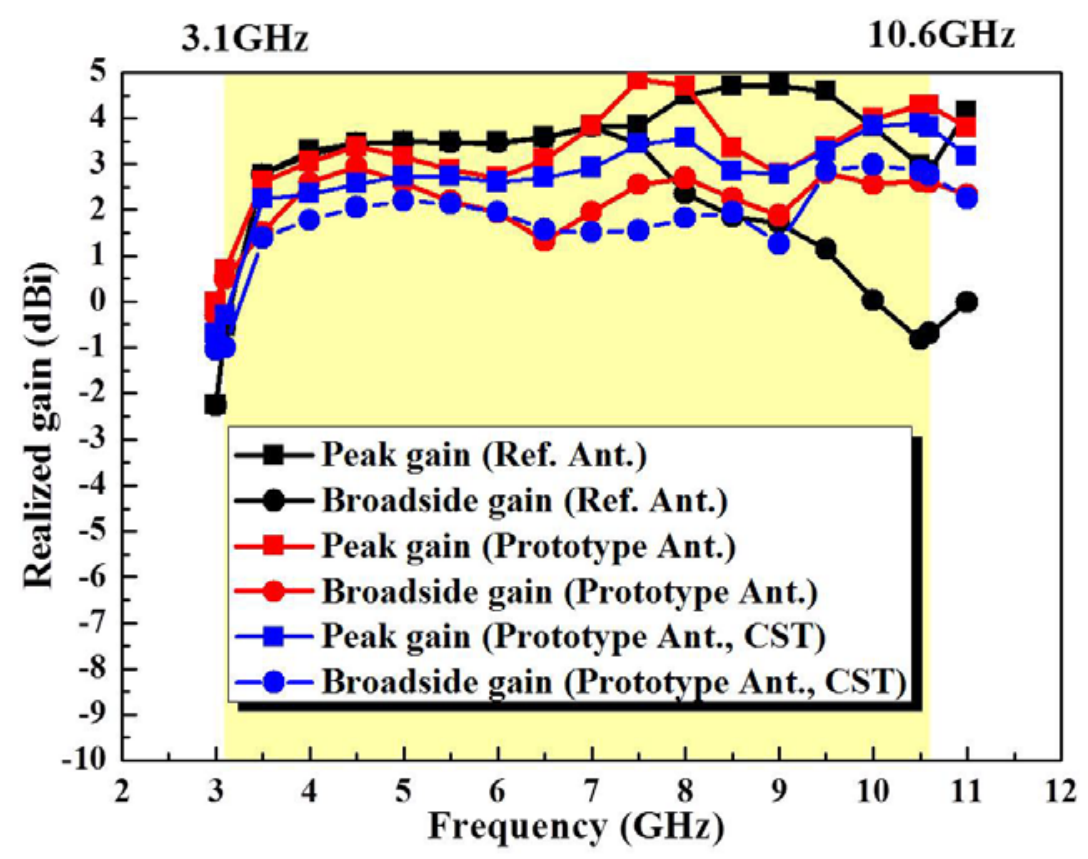

Figure 6. Surface current distributions on the half-elliptical monopole patch and the three NFRP strips at different frequencies.

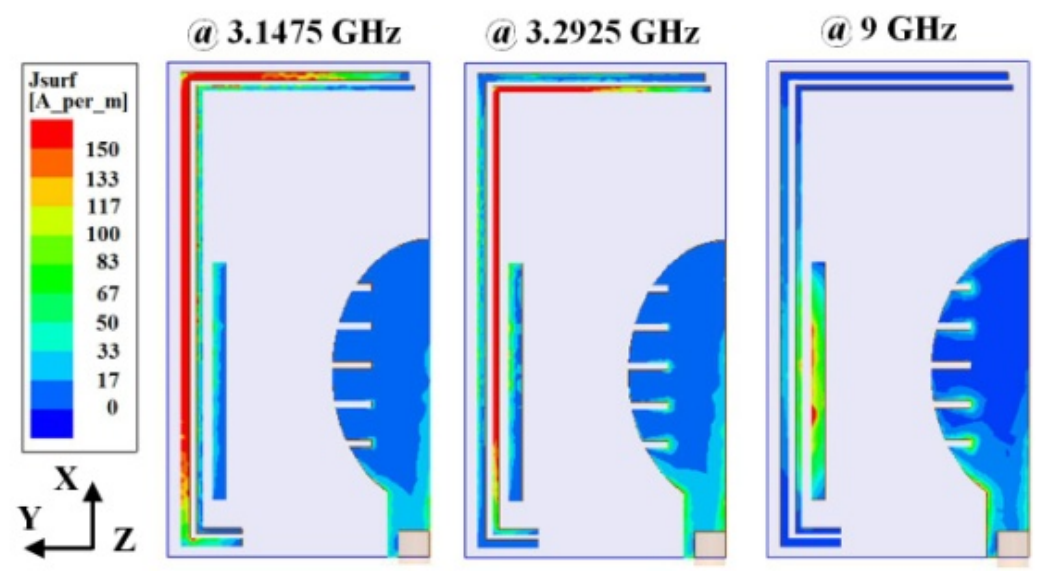


Figure 7. The impacts of the three NFRP strips on the VSWR of the prototype antenna as functions of the source frequency.

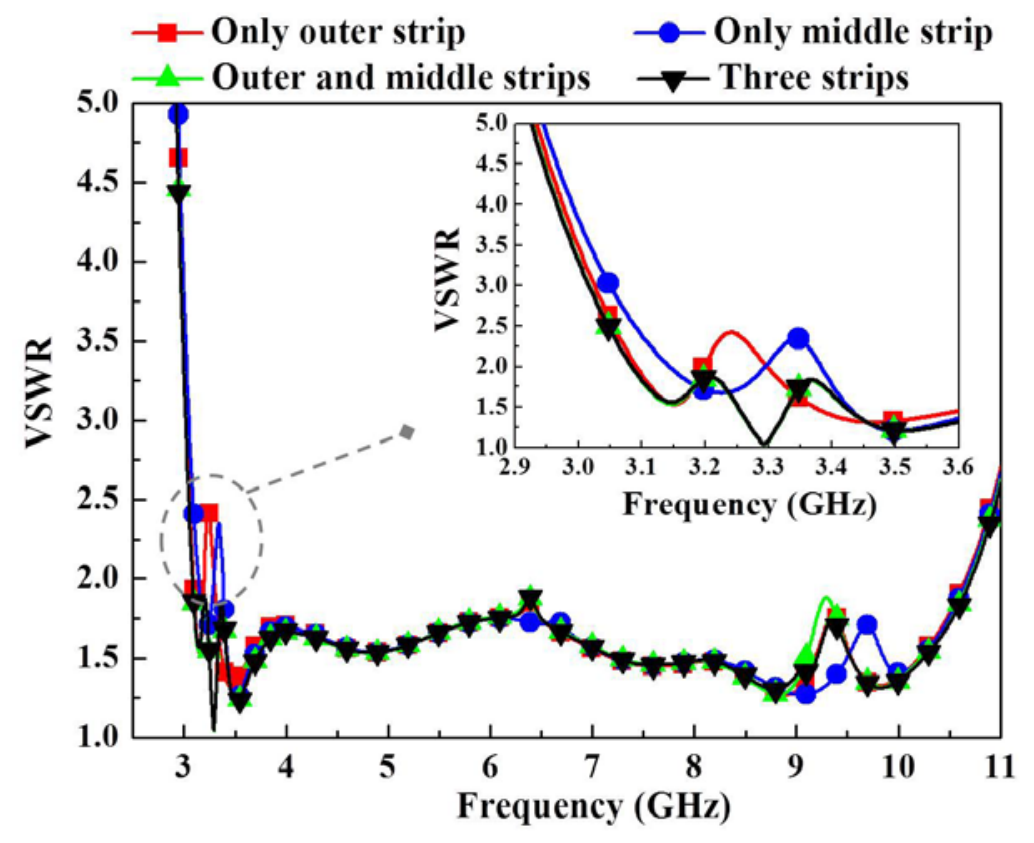


Figure 8. The VSWR of the prototype antenna as a function of the source frequency when the design parameters are varied. These parametric studies include: (a) the width of microstrip feed-line (W6), and the (b) length (L9) and (c) width (W7) of the slot on the ground strip.

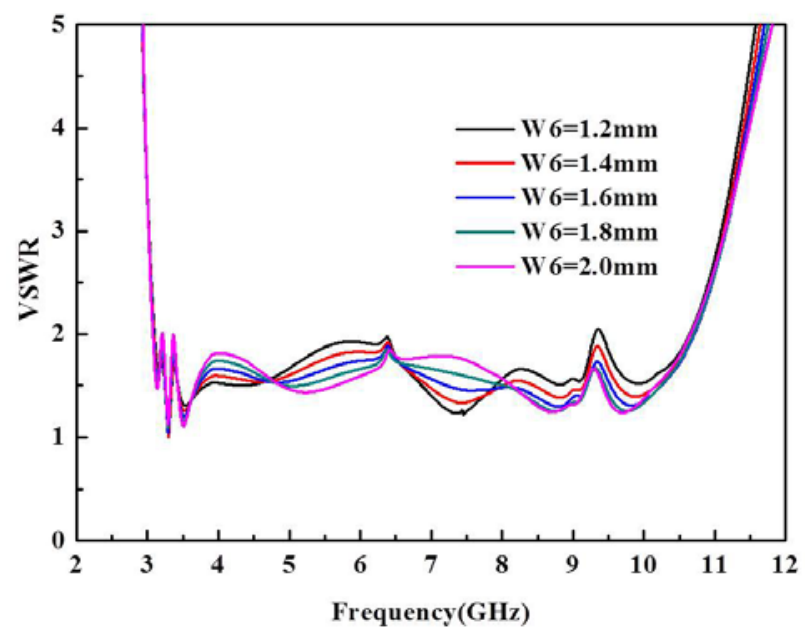

(a)

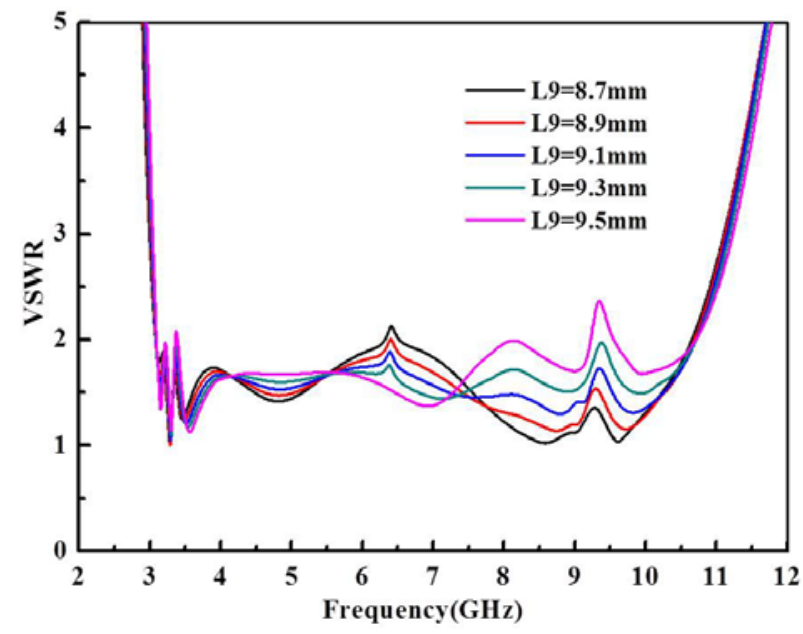

(b) 


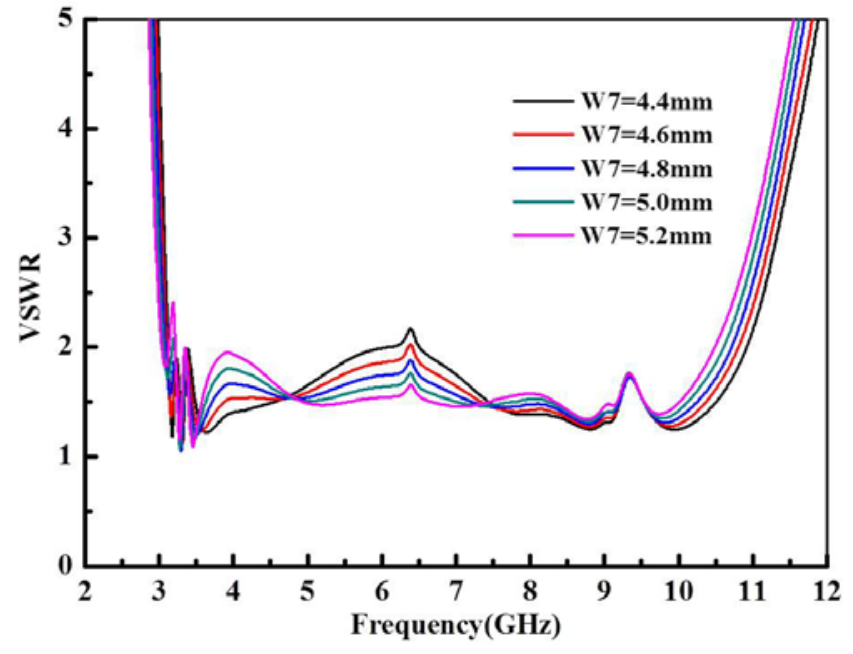

(c) 